\title{
Crystallite size distribution and dislocation structure determined by diffraction profile analysis: principles and practical application to cubic and hexagonal crystals
}

\author{
T. Ungár, J. Gubicza, G. Ribárik and A. Borbély
}

Copyright (C) International Union of Crystallography

Author(s) of this paper may load this reprint on their own web site provided that this cover page is retained. Republication of this article or its storage in electronic databases or the like is not permitted without prior permission in writing from the IUCr. 
Journal of

Applied

Crystallography

ISSN 0021-8898

Received 9 November 2000

Accepted 26 February 2001

\section{Crystallite size distribution and dislocation structure determined by diffraction profile analysis: principles and practical application to cubic and hexagonal crystals}

\author{
T. Ungár,* J. Gubicza, G. Ribárik and A. Borbély \\ Department of General Physics, Eötvös University, Budapest, PO Box 32, H-1518, Hungary. \\ Correspondence e-mail: ungar@ludens.elte.hu
}

\begin{abstract}
Two different methods of diffraction profile analysis are presented. In the first, the breadths and the first few Fourier coefficients of diffraction profiles are analysed by modified Williamson-Hall and Warren-Averbach procedures. A simple and pragmatic method is suggested to determine the crystallite size distribution in the presence of strain. In the second, the Fourier coefficients of the measured physical profiles are fitted by Fourier coefficients of well established $a b$ initio functions of size and strain profiles. In both procedures, strain anisotropy is rationalized by the dislocation model of the mean square strain. The procedures are applied and tested on a nanocrystalline powder of silicon nitride and a severely plastically deformed bulk copper specimen. The $\mathrm{X}$-ray crystallite size distributions are compared with size distributions obtained from transmission electron microscopy (TEM) micrographs. There is good agreement between X-ray and TEM data for nanocrystalline loose powders. In bulk materials, a deeper insight into the microstructure is needed to correlate the $\mathrm{X}$-ray and TEM results.
\end{abstract}

(C) 2001 International Union of Crystallography Printed in Great Britain - all rights reserved
Sen Gupta, 1999; Cheary et al., 2000). The g dependence is further complicated by strain anisotropy, which means that neither the breadth nor the Fourier coefficients of the diffraction profiles are monotonous functions of the diffraction angle or g (Caglioti et al., 1958; Ungár \& Borbély, 1996; Le Bail \& Jouanneaux, 1997; Dinnebier et al., 1999; Stephens, 1999; Scardi \& Leoni, 1999; Černý et al., 2000).

Peak profile analysis can only be successful if the strain effect is separated correctly. Two different models have been developed so far for strain anisotropy: (i) a phenomenological model based on the anisotropy of the elastic properties of crystals (Stephens, 1999) and (ii) the dislocation model (Ungár \& Borbély, 1996) based on the mean square strain of dislocated crystals (Krivoglaz, 1969, 1996; Wilkens, 1970a,b). The dislocation model of $\left\langle\varepsilon_{L, g}^{2}\right\rangle$ takes into account that the contribution of a dislocation to strain-induced broadening (strain broadening) of a diffraction profile depends on the relative orientations of the line and Burgers vectors of the dislocations and the diffraction vector, similar to the contrast effect of dislocations in electron microscopy. Anisotropic contrast can be summarized in contrast factors, $C$, which can be calculated numerically on the basis of the crystallography of dislocations and the elastic constants of the crystal (Wilkens, 1970a, 1987; Groma et al., 1988; Klimanek \& Kužel, 1988; Kužel \& Klimanek, 1988; Ungár \& Tichy, 1999; Ungár, Dragomir et al., 1999; Borbély et al., 2000; Cheary et al., 2000). By appropriate determination of the type of dislocations and 
Burgers vectors present in the crystal, the average contrast factors, $\bar{C}$, for the different Bragg reflections can be determined. Using the average contrast factors in the 'modified' Williamson-Hall plot and in the 'modified' Warren-Averbach procedure, the different averages of crystallite sizes, the density and the effective outer cut-off radius of dislocations can be obtained (Ungár \& Borbély, 1996; Ungár et al., 1998).

It can be shown that, once the strain contribution has been separated, diffraction peak profiles depend on the shape, the mean size and the size distribution of crystallites or coherently diffracting domains (Bertaut, 1950; Rao \& Houska, 1986; Langford et al., 2000). If the shape of the crystallites can be assumed to be uniform, the area- and volume-weighted mean crystallite sizes can be determined from the Fourier coefficients and the integral breadths of the X-ray diffraction profiles (Krill \& Birringer, 1998; Rand et al., 1993; Wilson, 1962; Gubicza et al., 2000). These two mean sizes of crystallites can be used for the determination of a crystallite size distribution function. Armstrong \& Kalceff (1999) have recently developed a method of maximum entropy for the determination of column length distribution from size-broadened profiles. There is a large amount of experimental evidence that the crystallite size distribution is usually log-normal (Krill \& Birringer, 1998; Terwilliger \& Chiang, 1995; Ungár, Borbély et al., 1999; Gubicza et al., 2000). Hinds (1982) proposed formulae to calculate the two characteristic parameters of the log-normal size distribution function from the area- and volume-weighted means. Krill \& Birringer (1998) determined the weighted mean crystallite sizes from the Fourier transform of X-ray diffraction profiles. Using the formulae of Hinds (1982), they calculated the parameters of the log-normal size distribution for nanocrystalline palladium. Langford et al. (2000) have elaborated a whole-powder-pattern fitting procedure to determine the crystallite size distribution in the absence of strain; they assumed spherical morphology and lognormal size distribution of crystallites and discussed the shape of profiles in terms of Lorentzian and Gaussian components depending on the variance of the size distribution.

The aim of this paper is to present two different procedures for the determination, from diffraction profiles, of the size distribution of crystallites in the presence of strain. Strain is given in terms of the dislocation density and arrangement. The first method uses the three apparent size parameters obtained from the FWHM, the integral breadths and the first few Fourier coefficients of the diffraction profiles using the modified Williamson-Hall and Warren-Averbach procedures. The measured apparent size parameters are matched, by the method of least squares, to the calculated values obtained from the theoretical size profile. In the second procedure, the Fourier transforms of the experimentally determined peak profiles are fitted by the Fourier coefficients of ab initio physical functions of the size and strain profiles. The only fitting parameters are the median and the variance of the size distribution function, the density and the arrangement of dislocations, and one or two parameters corresponding to the dislocation contrast factors in cubic or hexagonal crystals, respectively. In the present paper, the application and testing of the two procedures on the profiles of two representative materials, (a) a submicrometre-grain-size copper specimen deformed by equal-channel angular (ECA) pressing (Valiev et $a l ., 1994)$ and $(b)$ a hexagonal $\mathrm{Si}_{3} \mathrm{~N}_{4}$ nanocrystalline ceramic powder produced by nitridation of silicon and subsequent milling, are discussed. In both cases, TEM microstructures are analysed and discussed in parallel with the $\mathrm{X}$-ray peak profile analysis. The specific surface area of $\mathrm{Si}_{3} \mathrm{~N}_{4}$ nanopowder was additionally investigated by the method of BrunauerEmmett-Teller (BET) and is discussed together with the TEM and X-ray results.

\section{Diffraction profile analysis based on the widths and the first Fourier coefficients of profiles (WFFC)}

\subsection{The hierarchy of lattice defects}

Lattice defects fit into a simple hierarchy according to their strain fields: the strain fields of (i) point defects, (ii) linear defects or (iii) planar defects decay as $1 / r^{2}, 1 / r$ or are spaceindependently homogeneous, respectively, where $r$ is the distance from the defects. The three different types of spacial dependences are of short- and long-range order, and homogeneous, respectively. As a result of the reciprocity between crystal and reciprocal space, point defects have diffraction effects far from the fundamental Bragg reflections, often referred to as Huang scattering (Trinkaus, 1972). The strain fields of linear defects are of long-range character; therefore their diffraction effects cluster around the fundamental Bragg reflections. This is the diffraction effect known as diffraction peak broadening; the science related to it is peak profile (or line profile) analysis. The strain fields of planar defects are space independent or homogeneous; thus they cause lattice parameter changes or shifts of Bragg reflections. In reality, lattice defects are more complex and their effects on peak shape can be a mixture of the three well separated cases. Stacking faults, for example, can cause peak shifts and peak broadening simultaneously, since they are usually bounded by partial dislocations. Despite this complex behaviour, dislocations play a special and unique role: they are always present (a) either as the major component in complex lattice defects or (b) as the only lattice defects which distort the crystal lattice to such an extent that it becomes visible as profile (or line) broadening in a diffraction experiment. For this reason, in the present account we consider the effect of dislocations in strain broadening. The effect of stacking faults and/or planar defects, like grain boundaries, may be the subject of further developments (Scardi \& Leoni, 1999).

\subsection{The modified Williamson-Hall and Warren-Averbach procedures}

Within the kinematical theory of X-ray diffraction, the physical profile of a Bragg reflection is given by the convolution of the size and the distortion profiles (Warren, 1959; Wilson, 1962): 


$$
I^{P}=I^{S} * I^{D}
$$

where the superscripts $S$ and $D$ stand for size and distortion, respectively. The Fourier transform of this equation is known as the Warren-Averbach method (Warren, 1959):

$$
\ln A(L) \simeq \ln A_{L}^{S}-2 \pi^{2} L^{2} g^{2}\left\langle\varepsilon_{g, L}^{2}\right\rangle,
$$

where $A(L)$ are the absolute values of the Fourier coefficients of the physical profiles, $A_{L}^{S}$ are the size Fourier coefficients, $g$ is the absolute value of the diffraction vector and $\left\langle\varepsilon_{g, L}^{2}\right\rangle$ is the mean square strain. $L$ is the Fourier length defined as $L=n a_{3}$ (Warren, 1959), where $a_{3}=\lambda / 2\left(\sin \theta_{2}-\sin \theta_{1}\right), n$ are integers starting from zero, $\lambda$ is the wavelength of X-rays and $\left(\theta_{2}-\theta_{1}\right)$ is the angular range of the measured diffraction profile.

In a dislocated crystal, for small $L$ values, $\left\langle\varepsilon_{g, L}^{2}\right\rangle$ can be given as (Krivoglaz, 1969, 1996; Wilkens, 1970a,b)

$$
\left\langle\varepsilon_{g, L}^{2}\right\rangle \simeq\left(\rho \bar{C} b^{2} / 4 \pi\right) \ln \left(R_{e} / L\right),
$$

where $\rho, b$ and $R_{e}$ are the density, the modulus of the Burgers vector and the effective outer cut-off radius of dislocations, respectively. The peak broadening caused by a dislocation depends on the relative orientations between the Burgers and line vectors of the dislocation and the diffraction vector, $\mathbf{b}, \mathbf{l}$ and $\mathbf{g}$, respectively. This effect is taken into account by the dislocation contrast factors $C$ (Krivoglaz, 1969; Wilkens, 1970a,b; Kužel \& Klimanek, 1988; Ungár, Dragomir et al., 1999). In a texture-free polycrystal or if the Burgers vector population on the different slip systems is random, the $C$ factors can be averaged over the permutations of the $h k l$ indices (Ungár \& Tichy, 1999). In the present work, we deal with cases in which this averaging is legitimate; therefore, in equation (3) the average dislocation contrast factor $\bar{C}$ is used. Inserting equation (3) into (2), the 'modified' Warren-Averbach equation is obtained (Ungár \& Borbély, 1996):

$$
\ln A(L) \simeq \ln A^{S}(L)-\rho B L^{2} \ln \left(R_{e} / L\right)\left(K^{2} \bar{C}\right)+O\left(K^{4} \bar{C}^{2}\right),
$$

where $K=2 \sin \theta / \lambda$ and $K=\mathbf{g}$ at the exact Bragg position, $B=$ $\pi b^{2} / 2$ and $O$ stands for higher order terms in $K^{2} \bar{C}$. The size parameter corresponding to the Fourier coefficients is denoted by $L_{0}$. It is obtained from the size Fourier coefficients, $A^{S}$, by taking the intercept of the initial slope at $A^{S}=0$ (Warren, 1959) and it gives the area-weighted mean column length (Guinier, 1963).

Based on the dislocation model of strain, the FWHM and the integral breadths of profiles can be evaluated by the modified Williamson-Hall plot ( $c f$. Ungár \& Borbély, 1996). In the following, it will be shown that if this plot is given as a function of $K \bar{C}^{1 / 2}$, then it has to start with a horizontal slope. The only $h k l$-dependent term in $\left\langle\varepsilon_{g, L}^{2}\right\rangle$ is $\bar{C}$, which can be separated from the $L$-dependent part as

$$
\left\langle\varepsilon_{g, L}^{2}\right\rangle=\left\langle\varepsilon_{L}^{2}\right\rangle \bar{C} .
$$

Using equations (2) and (5) and assuming that the Fourier coefficients are normalized to unity at $L=0$, the integral breadth of a profile can be given as

$$
\Delta K_{\beta}=\left\{\int_{0}^{\infty} 2 A^{S}(L) \exp \left[-2 \pi^{2} L^{2}\left\langle\varepsilon_{L}^{2}\right\rangle\left(K \bar{C}^{1 / 2}\right)^{2}\right] \mathrm{d} L\right\}^{-1} .
$$

In the present work it is assumed that the main source of strain is dislocations. The best solution of $\left\langle\varepsilon_{L}^{2}\right\rangle$ for dislocations in the entire $L$ range has been given, so far, by Wilkens (1970b) and is discussed in $\$ 3.1$ below.

Let us introduce the notation $z=K \bar{C}^{1 / 2}$. Since the integrand in (6) is an analytic function of $z$ for any fixed value of $L$, the integral in (6) is also analytic for $z$. Since, further, the integral at $z=0$ is non-zero, its reciprocal, i.e. $\Delta K_{\beta}$, is also analytic at $z$ $=0$. This means that $\Delta K_{\beta}$ can be developed into a power series of $z$ around zero:

$$
\Delta K_{\beta}(z)=\sum_{n=0}^{\infty} a_{n} z^{n}
$$

Since $\Delta K_{\beta}$ is an even function of $z$,

$$
\Delta K_{\beta}(z)=\Delta K_{\beta}(-z)=\sum_{n=0}^{\infty}(-1)^{n} a_{n} z^{n} .
$$

From $(7 a)$ and $(7 b)$, for each $n, a_{n}=(-1)^{n} a_{n}$, from which it follows that for the odd values of $n, a_{n}=0$. In particular,

$$
\left.\frac{\mathrm{d} \Delta K_{\beta}}{\mathrm{d} z}\right|_{z=0}=0 \text {. }
$$

This means that the modified Williamson-Hall plot of the integral breadths starts with a zero slope and has the following form:

$$
\Delta K_{\beta}=1 / d+\alpha\left(K \bar{C}^{1 / 2}\right)^{2}+O\left(K \bar{C}^{1 / 2}\right)^{4} .
$$

The FWHM can also be shown to have the same $z$ behaviour:

$$
\Delta K_{\mathrm{FWHM}}=0.9 / D+\alpha^{\prime}\left(K \bar{C}^{1 / 2}\right)^{2}+O\left(K \bar{C}^{1 / 2}\right)^{4},
$$

where $d$ and $D$ are the apparent size parameters corresponding to the integral breadth and the FWHM. They are obtained by extrapolation to $K=0$ in the usual manner (Ungár $\&$ Borbély, 1996). $d$ provides the volume-weighted mean column length of the crystallites in the specimen (Guinier, 1963; Langford et al., 2000). The fourth-order terms in $z$, i.e. $O\left(K \bar{C}^{1 / 2}\right)^{4}$, are usually small compared to the first two terms in the power series in (8) and (9).

In a few previous papers, in which the modified WilliamsonHall procedure has been suggested and applied to evaluate apparent size values, the experimental data have already indicated the type of $z$ behaviour derived in equations (8) and (9) (Ungár \& Borbély, 1996; Ungár et al., 1998; Ungár, Dragomir et al., 1999; Ungár \& Tichy, 1999; Ungár, Leoni \& Scardi, 1999; Gubicza et al., 2000). If strain is not mainly caused by dislocations, deviations from purely quadratic $K$ dependence of $\Delta K$ may also be anticipated. For example, if strain distribution is Gaussian, i.e. the displacement of atoms from their equilibrium positions is strictly random, then the mean square strain becomes constant (Warren, 1959). In such a case, $\Delta K$ is a linear function of $K$.

\subsection{The average dislocation contrast factors}

The average dislocation contrast factors are the weighted average of the individual $C$ factors either over the dislocation population or over the permutations of the $h k l$ indices 
(Krivoglaz, 1969; Wilkens, 1970a,b; Kužel \& Klimanek, 1988). Based on the theory of line broadening caused by dislocations, it can been shown that in an untextured cubic and a hexagonal polycrystalline specimen, the values of $\bar{C}$ are simple functions of the invariants of the fourth-order polynomials of $h \mathrm{kl}$ (Ungár \& Tichy, 1999):

$$
\bar{C}=\bar{C}_{h 00}\left(1-q H^{2}\right)
$$

and

$$
\bar{C}=\bar{C}_{h k 0}\left(1+\frac{\left\{A\left[h^{2}+k^{2}+(h+k)^{2}\right]+B l^{2}\right\} l^{2}}{\left[h^{2}+k^{2}+(h+k)^{2}+(3 / 2)(a / c)^{2} l^{2}\right]^{2}}\right),
$$

respectively, where $\bar{C}_{h 00}$ and $\bar{C}_{h k 0}$ are the average dislocation contrast factors for the $h 00$ and $h k 0$ reflections, respectively, $H^{2}=\left(h^{2} k^{2}+h^{2} l^{2}+k^{2} l^{2}\right) /\left(h^{2}+k^{2}+l^{2}\right)^{2} ; q, A$ and $B$ are parameters depending on the elastic constants and on the character of dislocations (e.g. edge or screw type) in the crystal and $c / a$ is the ratio of the two lattice constants of the hexagonal crystal. It is worth noting that the fourth-order invariants of the $h k l$ indices appear also in the more phenomenological description of anisotropic strain broadening as presented by Stephens (1999) and Popa (1998).

\subsection{Determination of the size distribution of crystallites}

Three size parameters were determined by the modified Williamson-Hall and Warren-Averbach procedures: $D$ from the FWHM, $d$ from the integral breadths and $L_{0}$ from the Fourier coefficients. A pragmatic and self-consistent numerical procedure has been worked out to relate the experimental $D, d$ and $L_{0}$ values to the parameters of a crystallite size distribution density function $f(x)$. It has been observed by many authors that the size distribution of crystallites in powder or bulk specimens is log-normal (cf. Langford et al., 2000). This is especially true in plastically deformed bulk or in nanocrystalline materials (Krill \& Birringer, 1998; Terwilliger \& Chiang, 1995; Ungár, Borbély et al., 1999; Valiev et al., 2000):

$$
f(x)=\left[1 /(2 \pi)^{1 / 2} \sigma\right](1 / x) \exp \left\{-[\ln (x / m)]^{2} / 2 \sigma^{2}\right\},
$$

where $x$ is the size of a crystallite from the size distribution, $\sigma^{2}$ is the variance and $m$ is the median of the size distribution function (Langford et al., 2000). Guinier (1963) has shown that if the crystallite is distortion-free, the Bragg peak profile can be described as [Guinier, 1963, equation (5.18) therein]:

$$
I^{S}(s)=\int_{0}^{\infty}\left[\sin ^{2}(\pi \mu s) / \mu(\pi s)^{2}\right] g(\mu) \mathrm{d} \mu,
$$

where $s=\Delta(2 \theta) / \lambda, \mu$ is the column length and $g(\mu) \mathrm{d} \mu$ represents the volume fraction of the columns for which the length parallel to the diffraction vector lies between $\mu$ and $\mu+$ $\mathrm{d} \mu$. The relationship between $g(\mu)$ and $f(x)$ depends on the shape of the crystallites, since the volume fraction of the column lengths in a given crystallite is related to its geometrical boundaries. For spherical crystallites, the relationship between $g(\mu)$ and $f(x)$ can be given in the following form:

$$
g(\mu)=N \mu^{2} \int_{\mu}^{\infty} f(x) \mathrm{d} x,
$$

where $N$ is a normalization factor. Substituting equation (12) into (14), calculating the integral in equation (14) and substituting (14) into (13), the intensity distribution corresponding to size broadening is obtained as

$$
I^{S}(s)=\int_{0}^{\infty} \mu\left[\sin ^{2}(\pi \mu s) 2(\pi s)^{2}\right] \operatorname{erfc}\left\{[\ln (\mu / m)] / 2^{1 / 2} \sigma\right\} \mathrm{d} \mu,
$$

where erfc is the complementary error function. It can be seen from equation (15) that the shape of the peak profile depends only on $\sigma$ and $m$. [Here we note that (15) gives only the intensity distribution without normalizing with respect to either the maximum or the integral of the intensity.] The theoretical function $I^{S}(s)$ can provide numerically calculated apparent size parameters corresponding to its FWHM, integral breadth and Fourier coefficients, denoted by $D_{\sigma, m}, d_{\sigma, m}$ and $L_{0}^{\sigma, m}$, respectively. The median and the variance of the size distribution function are obtained by the method of leastsquares fitting:

$$
\left(D_{\sigma, m}-D\right)^{2}+\left(d_{\sigma, m}-d\right)^{2}+\left(L_{0}^{\sigma, m}-L_{0}\right)^{2}=\text { minimum, }
$$

in which the fitting is carried out by varying $\sigma$ and $m$.

The main advantages of this method are the following: (i) the procedure uses three experimental apparent size parameters to determine the two parameters of the size distribution function, thus decreasing the errors introduced by experimental uncertainties; (ii) if one of the three size parameters cannot be determined, the two remaining are enough to calculate $\sigma$ and $m$ by this procedure; (iii) the method outlined above can also be applied if the size distribution is different from log-normal by inserting the appropriate function into equation (14). For spherical crystallites with lognormal size distribution, the area-, volume- and arithmetically weighted mean crystallite sizes are obtained as (Hinds, 1982)

$$
\begin{aligned}
\langle x\rangle_{\text {area }} & =m \exp \left(2.5 \sigma^{2}\right), \\
\langle x\rangle_{\mathrm{vol}} & =m \exp \left(3.5 \sigma^{2}\right)
\end{aligned}
$$

and

$$
\langle x\rangle_{\text {arithm }}=m \exp \left(0.5 \sigma^{2}\right),
$$

respectively. The procedure has also been worked out for ellipsoidal disc-shape crystallites, enabling the determination of the size distribution functions of crystallites with nonspherical shape (Ungár et al., 2001).

\section{Whole-profile fitting by the Fourier coefficients of $a b$ initio size and strain functions (WPFC)}

The fundamental equations of diffraction profile analysis are equations (1) and (2), which tell us that the size and strain profiles are in convolution and the Fourier coefficients are in product. Once we know these functions, it is only a question of skillful numerical calculus to make a fitting between experiment and theory. The size profile for spherical crystallites 
having a log-normal size distribution is given by equation (15). Here we show that using the strain profile suggested by Wilkens $(1970 a, b)$ for dislocated crystals, the experimental profiles can be fitted by ab initio physical functions for both size and strain broadening, respectively. We show further that the fitting procedure provides well established physical parameters characterizing the microstructure, which can be compared with parameters obtained by other methods, especially TEM, in a straightforward manner. It should be noted that the size profile function for similar crystallite shapes and size distributions has recently been derived by Langford et al. (2000). Because of a different kind of derivation and summation, their formula [equation (21) of Langford et al., 2000] is different from equation (15) herein; however, the two equations are mathematically equivalent.

\subsection{The strain profile for dislocations}

In equation (3), it has been shown that for small $L$ values, the mean square strain for dislocations is described by a logarithmic function. Wilkens evaluated $\left\langle\varepsilon_{g, L}^{2}\right\rangle$ in the entire range of $L$ for screw dislocations (Wilkens, 1970a,b). For the following reasons this will be used as the ab initio function for strain broadening: (i) this is probably the best available expression of $\left\langle\varepsilon_{g, L}^{2}\right\rangle$ for dislocations (Levine \& Thomson, 1997; Groma, 1998); (ii) experiments on plastically deformed copper single crystals (Wilkens, 1988), and (iii) computer simulations (Kamminga \& Delhez, 2000) have shown that it works even for edge dislocations. The detailed expression of $\left\langle\varepsilon_{g, L}^{2}\right\rangle$ given by Wilkens is (Wilkens, $1970 \mathrm{~b}$, equations A.6 to A. 8 therein)

$$
\left\langle\varepsilon_{g, L}^{2}\right\rangle=(b / 2 \pi)^{2} \pi \rho C f(\eta),
$$

where

$$
\eta=(1 / 2) \exp (-1 / 4)\left(L / R_{e}^{\prime}\right)
$$

In the following we call $f(\eta)$ the Wilkens function. For $\eta \leq 1$,

$$
\begin{aligned}
f(\eta)= & -\ln \eta+(7 / 4-\ln 2)+512 / 90 \pi \eta \\
& +(2 / \pi)\left(1-1 / 4 \eta^{2}\right) \int_{0}^{\eta}(\arcsin V) / V \mathrm{~d} V \\
& -(1 / \pi)\left(769 / 180 \eta+41 \eta / 90+2 \eta^{3} / 90\right)\left(1-\eta^{2}\right)^{1 / 2} \\
& -(1 / \pi)\left(11 / 12 \eta^{2}+7 / 2+\eta^{2} / 3\right) \arcsin \eta+\eta^{2} / 6,
\end{aligned}
$$

and for $\eta \geq 1$,

$$
f(\eta)=512 / 90 \pi \eta-[11 / 24+(1 / 4) \ln 2 \eta]\left(1 / \eta^{2}\right) .
$$

It can be seen that the Wilkens function has a logarithmic singularity at small $\eta$ values and decays as a hyperbola for large values of $\eta$. In the numerical calculations, $f(\eta)$ has been applied as it stands in equations (22) and (23) with the exception that the integral in (22) has been approximated by a series expansion. Here we note that, strictly speaking, the Wilkens function was calculated assuming screw dislocations; however, Kamminga \& Delhez (2000) have shown recently that the calculations remain valid for edge dislocations.
According to equations (2) and (20), the Fourier coefficients of the strain profile can be given as

$$
A_{g}^{D}(L)=\exp \left[-\rho B L^{2} f(\eta) g^{2} \bar{C}\right] .
$$

In accordance with definitions used in previous works (Levine \& Thomson, 1997; Groma, 1998; Ungár et al., 1982; Hecker et al., 1997; Zehetbauer et al., 1999; Ungár \& Borbély, 1996; Ungár \& Tichy, 1999), the effective outer cut-off radius of dislocations, $R_{e}$, will be considered as defined in equation (3). $R_{e}$ and $R_{e}^{\prime}$ are related as

$$
R_{e}=\exp (2) R_{e}^{\prime}=7.39 R_{e}^{\prime} .
$$

It is physically more appropriate to use the dimensionless parameter

$$
M=R_{e} \rho^{1 / 2}
$$

defined by Wilkens as the dislocation arrangement parameter (Wilkens, 1970b). The value of $M$ gives the strength of the dipole character of dislocations: if $M$ is small or large, the dipole character and the screening of the displacement field of dislocations are strong or weak, respectively. At the same time, strong or weak screening and small or large values of $M$ mean strong or weak correlation in the dislocation distributions and long or short tails in the diffraction profiles, respectively. Long or short tails of the diffraction profiles mean that the tail parts of the profiles are close to Lorentzian or Gaussian type functions, respectively; however, they are never exactly identical to either of the two simple functions (Ungár et al., 1982; Ungár \& Tichy, 1999; Wilkens, 1987). This also means that when diffraction profiles reveal similarity to either of the two shapes, i.e. Lorentzian or Gaussian, it is more appropriate if this behaviour is interpreted by concomitant size and strain broadening (Barabash \& Klimanek, 1999; Langford, et al., 2000).

\subsection{The Fourier transform of size profile}

The Fourier transform of the size intensity function $I^{S}$ in equation (15) yields the following formula:

$$
A^{S}(L) \simeq \int_{|L|}^{\infty}\left(\mu^{2}-|L| \mu\right) \operatorname{erfc}\left\{[\ln (\mu / m)] / 2^{1 / 2} \sigma\right\} \mathrm{d} \mu .
$$

Calculating the integral in equation (26), the Fourier coefficients of the size profile can be obtained in closed form as a function of the two parameters of the log-normal size distribution, $m$ and $\sigma$ :

$$
\begin{aligned}
A^{S}(L) \simeq & {\left[m^{3} \exp \left(4.5 \sigma^{2}\right) / 3\right] \operatorname{erfc}\left\{[\ln (|L| / m)] / 2^{1 / 2} \sigma-1.5\left(2^{1 / 2}\right) \sigma\right\} } \\
& -\left[m^{2} \exp \left(2 \sigma^{2}\right)|L| / 2\right] \operatorname{erfc}\left\{[\ln (|L| / m)] / 2^{1 / 2} \sigma-2^{1 / 2} \sigma\right\} \\
& +\left(|L|^{3} / 6\right) \operatorname{erfc}\left\{[\ln (|L| / m)] / 2^{1 / 2} \sigma\right\} .
\end{aligned}
$$

\subsection{Fitting procedure}

A numerical procedure has been worked out for fitting the Fourier transform of the experimental profiles by the theoretical functions of size and strain Fourier transforms given by 
equations (24) and (27). The fitting of the Fourier transform instead of the intensity profile is performed for the following reasons: $(a)$ the size and the strain profiles are in convolution [see equation (1)]; therefore it is more convenient to work on the Fourier coefficients which are in product; $(b)$ the size and strain Fourier coefficients are given in explicit forms [see equations (24) and (27)]; (c) the instrumental correction can be easily carried out on the Fourier coefficients using complex division, as in the Stokes correction (Stokes, 1948). The numerical procedure has the following steps. (i) The Fourier coefficients of the measured physical profiles are calculated by a non-equidistantly sampling Fourier transformation (NESFT). (ii) The Fourier coefficients of the size and strain profiles are calculated by using equations (22), (23), (24), (27) and (10) or (11) by expanding the integral in equation (22) into a Taylor series. (iii) The experimental and the calculated Fourier coefficients are compared by the Marquardt-Levenberg non-linear least-squares procedure using a modified version of the GNUPLOT program package (for the original GNUPLOT program package see http://www.gnuplot.org).

The whole profile fitting procedure is based on five or six fitting parameters for cubic or hexagonal crystals, respectively: (i) $m$ and (ii) $\sigma$ of the log-normal size distribution function (assuming spherical crystallites), (iii) $\rho$ and (iv) $M$ in the strain profile [see equations (20), (22) and (23)], and (v) $q$, or $A$ and $B$, for the average dislocation contrast factors in cubic, or hexagonal, crystals, respectively. The quality of the fitting is measured by the sum of the squares of the differences between the calculated and the input Fourier coefficients: SSR (sum of squared residuals). Further details of the fitting procedure and the fitting program may be found elsewhere (Ribárik et al., 2001).

\subsection{Boundary conditions and assumptions}

These procedures assume that (i) strain is caused by dislocations, (ii) either the specimen is a texture-free polycrystal, or it is a random powder, or the Burgers vector population in the possible slip systems is random, (iii) the crystallite size distribution is lognormal, and (iv) the shape of the diffraction domains is spherical. If there is evidence that the microstructure contains other lattice defects, e.g. stacking faults, the evaluation procedures should be corrected, which increases the number of fitting parameters. If the assumption (ii) is not true, then equations (10) and (11) cannot be used for the average dislocation contrast factors and the individual contrast factors calculated numerically should be used as fixed parameters, either for different reflections or for the different components of an $h \mathrm{kl}$ reflection. If the crystallite size distribution is not log-normal, but has analytical form with two free parameters, then equations (15), (17), (18), (19), (26) and (27) have to be recalculated, but the procedures and the number of fitting parameters are not changed. The deviation of the shape of crystallites from spherical would increase the number of fitting parameters in the modified Williamson-Hall plot and the modified Warren-Averbach method, and also in the whole-profile fitting procedure. In this case, the anisotropic broadening of diffraction profiles is caused by both the shape and the strain of the crystallites (Ungár et al., 2001).

As mentioned before, if the specimen is either a texturefree polycrystal or a powder, or the Burgers vector population in the possible slip systems is random, then the average contrast factors are given by equations (10) and (11). In this case, the parameters $q$, or $A$ and $B$, of the contrast factors can be obtained either by the whole-profile fitting procedure as described in $\$ 3.3$, or from the modified Williamson-Hall plot. In the latter case, equations (10) and (11) are inserted into (9), which can be solved for $D, \alpha^{\prime}$ and $q$, or $D, \alpha^{\prime}, A$ and $B$, for cubic or hexagonal crystals, respectively, by the method of least squares. However, the length of the Burgers vector, $b$, and the average dislocation contrast factors for the $h 00$ or $h k 0$ reflections, $\bar{C}_{h 00}$ or $\bar{C}_{h k 0}$, are input parameters of the evaluation methods for cubic or hexagonal crystals, respectively. For the calculation of these parameters, some information about the dislocation structure existing in the sample is necessary. Effective ways to find out the dominant dislocation slip system are transmission electron microscopy (TEM) measurements or the evaluation of the anisotropic strain broadening of diffraction profiles by the modified Williamson-Hall method (Ungár, Dragomir et al., 1999; Gubicza et al., 2001).

\section{Experimental}

\subsection{Samples}

A copper and a silicon nitride sample having cubic and hexagonal crystal structures, respectively, were investigated. A 99.98\% copper specimen (kindly provided by Professor R. Valiev), of about a few micrometres initial crystallite size, was produced by extrusion. The extruded sample was further deformed by ECA (equal-channel angular pressing) producing sub-micrometre average crystallite size (Valiev et al., 1994, 2000). In order to avoid machining effects, an approximately $100 \mu \mathrm{m}$ surface layer was removed from the specimen surface by chemical etching before the X-ray experiments. The silicon nitride sample investigated here was a commercial powder produced by nitridation of silicon and post-milling (powder LC12 from Starck Ltd, Germany). The X-ray phase analysis showed that the silicon nitride ceramic powder contained 97 vol. $\% \alpha-\mathrm{Si}_{3} \mathrm{~N}_{4}$ and 3 vol. $\% \beta$ - $\mathrm{Si}_{3} \mathrm{~N}_{4}$; therefore the microstructural parameters calculated for the major $\alpha-\mathrm{Si}_{3} \mathrm{~N}_{4}$ phase were taken as characteristic parameters for the entire powder.

\subsection{X-ray diffraction technique}

The diffraction profiles were measured by a special doublecrystal diffractometer with negligible instrument-induced broadening (Wilkens \& Eckert, 1964). A fine-focus rotating cobalt anode (Nonius FR 591) was operated as a line focus at $36 \mathrm{kV}$ and $50 \mathrm{~mA}(\lambda=0.1789 \mathrm{~nm})$. The symmetrical 220 reflection of a Ge monochromator was used for wavelength compensation at the position of the detector. The $K \alpha_{2}$ component of the Co radiation was eliminated by a $0.16 \mathrm{~mm}$ slit between the source and the Ge crystal. The profiles were registered by a linear position-sensitive gas-flow detector 
(OED 50 Braun, Munich). In order to avoid air scattering and absorption, the distance between the specimen and the detector was bridged by an evacuated tube closed by Mylar windows.

\subsection{Corrections for instrumental effects, background and overlapping peaks}

Instrumental corrections have not been performed in the present case since the X-ray diffraction measurements were carried out by a special double-crystal diffractometer with negligible instrumental broadening (see $\$ 4.2$ ). If, however, the instrumental effect could not be neglected, the observed line profile would be the convolution of the physical and the instrumental profiles. In this case, the function fitted to the Fourier transform of the observed profiles would be the product of the size, the strain and the instrumental Fourier coefficients, as in a usual Stokes correction (Stokes, 1948). The profiles were measured by a linear position-sensitive detector in 2048 channels, from which 900 to 1200 were used in the evaluation of each profile. The tails of the diffraction profiles were measured down to $10^{-3}$ or better in the special highresolution diffractometer with negligible instrumental broadening. Thus truncation affects the profiles only in the range where relative intensities are below $10^{-3}$ to $10^{-4}$. From each measured profile, about 250 to 500 Fourier coefficients were calculated in the case of the WPFC procedure. More details about the numerical procedures will be published in a separate paper (Ribárik et al., 2001).

If the profiles overlap, they have to be separated since the present evaluation methods are designed for individual profiles. Background subtraction and the separation of overlapping peaks are carried out in one step. Two analytical functions, usually a pair of Pearson VII functions or a pair of pseudo-Voigt functions, plus a linear background are fitted to the overlapping peaks. In the next step, one of the fitted peaks together with the linear background is subtracted, leaving the other peak free of overlap and background. The counterpart of the two overlapping peaks is obtained by changing the assignment of one of the peaks as 'background'. Practice has shown that neither the Pearson VII nor the pseudo-Voigt function is able to fit the physical profiles satisfactorily from the maxima down to the tails. The software enables the height of the linear background to be changed manually in an interactive mode. This was necessary because of the unsatisfactory fitting of the profile tails by the two analytical functions. The separated profiles are taken as individual diffraction profiles in the evaluation procedures. The separation procedure can be avoided by further improvement of the whole-profile fitting by Fourier coefficients (WPFC), in which the theoretical intensity function is produced by the inverse Fourier transformation of the theoretical Fourier transforms and fitted to the overlapping experimental intensity profiles. This improvement of the WPFC method, with $a b$ initio physical profile functions including strain, is under construction.

\subsection{Transmission electron microscopy and the measurement of specific surface area}

Transmission electron microscopy (TEM, Jeol JEM200CX instrument) has been used for direct measurement of the size distribution of crystallites. Bright-field images were used to measure the crystallite size in the samples. The specific surface area of the silicon nitride ceramic powder was determined from the nitrogen adsorption isotherms by the BET (Brunauer-Emmett-Teller) method (Lippenca \& Hermanns, 1961). Assuming that the particles have spherical shape, the area-weighted average particle size $(t)$ in nanometres was calculated as $t=6000 / q S$ where $q$ is the density in $\mathrm{g} \mathrm{cm}^{-3}$ and $S$ is the specific surface area in $\mathrm{m}^{2} \mathrm{~g}^{-1}$.

\section{Results and discussion}

\subsection{Microstructural parameters obtained by the method of widths and first Fourier coefficients (WFFC)}

Strain anisotropy is clearly seen in the conventional Williamson-Hall plot (Williamson \& Hall, 1953) of the FWHM and the integral breadths for copper, as shown by Ungár \& Borbély (1996). The FWHM and the integral breadths for copper are shown in a modified Williamson-Hall plot in Fig. 1. It can be seen that the measured data follow smooth curves. Similar plots can be constructed for the silicon nitride specimen. Using equation (10) or (11), equation (9) was solved for $D, \alpha^{\prime}$ and $q$ for cubic copper, or $D, \alpha^{\prime}, A$ and $B$ for hexagonal silicon nitride by the method of least squares. For the copper specimen, $q=1.90$ (3) (uncertainty within parentheses) has been obtained. In a previous work, the values of $q$ have been calculated for the most common dislocation slip system in copper with the Burgers vector $\mathbf{b}=$ $a / 2\langle 110\rangle$ (Ungár, Dragomir et al., 1999). It was found that for pure screw or pure edge dislocations, the values of $q$ are 2.37 or 1.68 , respectively. The experimental value obtained in the

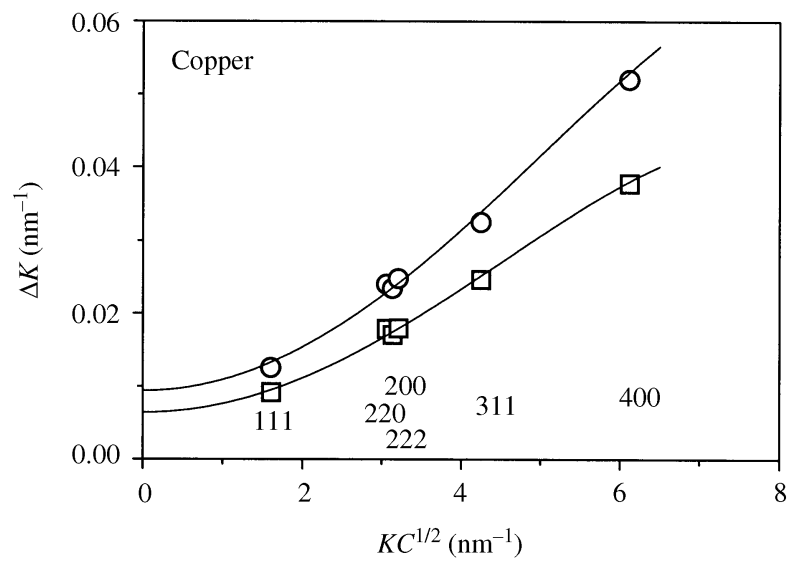

Figure 1

The modified Williamson-Hall plot of the FWHM (squares) and the integral breadths (circles) for copper deformed by equal-channel angular pressing (Valiev et al., 1994). The indices of reflections are also indicated. Note that $C$ is a function of $h k l$; see equation (10). 
present case is somewhat below the arithmetic average of the two limiting values of $q$. From this we conclude that the character of the prevailing dislocations is more edge than screw. This is in good agreement with other theoretical and experimental observations, according to which, in face-centred cubic (f.c.c.) metals during large deformations at low temperatures, screw dislocations annihilate more effectively than edge dislocations (Zehetbauer, 1993; Zehetbauer \& Seumer, 1993; Valiev et al., 1994). The value of $\bar{C}_{h 00}$ was determined in accordance with the experimental values of $q$ : $\bar{C}_{h 00}=0.30$ (1) (Ungár, Dragomir et al., 1999).

The $A$ and $B$ parameters in the contrast factors of silicon nitride were obtained from the modified Williamson-Hall plot as $A=3.33$ and $B=-1.78$. The value of $c / a$ was taken as 0.7150 . The value of $\bar{C}_{h k 0}$ was calculated numerically assuming elastic isotropy since, to the best knowledge of the authors, the anisotropic elastic constants of this material are not available. The isotropic $\bar{C}_{h k 0}$ factor was evaluated for the most commonly observed dislocation slip system in silicon nitride (Wang et al., 1996): $\langle 0001\rangle\{10 \overline{1} 0\}$. Taking 0.24 as the value of the Poisson ratio (Rajan \& Sajgalik, 1997), $\bar{C}_{h k 0}=0.0279$ was obtained. The best contrast factors corresponding to the integral breadths (also in the modified Williamson-Hall plot) and to the Fourier coefficients in the modified Warren-Averbach plot, were identical, within experimental error, to those obtained from the FWHM for both copper and silicon nitride. The quadratic regressions to the FWHM and the integral breadths give $D=140 \mathrm{~nm}$ and $d=106 \mathrm{~nm}$ for copper and $D=$ $74 \mathrm{~nm}$ and $d=57 \mathrm{~nm}$ for silicon nitride.

A typical plot according to the modified Warren-Averbach procedure is shown in Fig. 2 for the copper specimen. From the quadratic regressions, the size coefficients, $A^{S}$, were determined. The intersection of the initial slope at $A^{S}(L)=0$ yields the area-weighted average column length: $L_{0}=75$ and $41 \mathrm{~nm}$ for copper and $\mathrm{Si}_{3} \mathrm{~N}_{4}$, respectively. The dislocation densities obtained by using equation (4) are $1.6 \times 10^{15}$ and 7.7 $\times 10^{14} \mathrm{~m}^{-2}$ for copper and silicon nitride, respectively. The

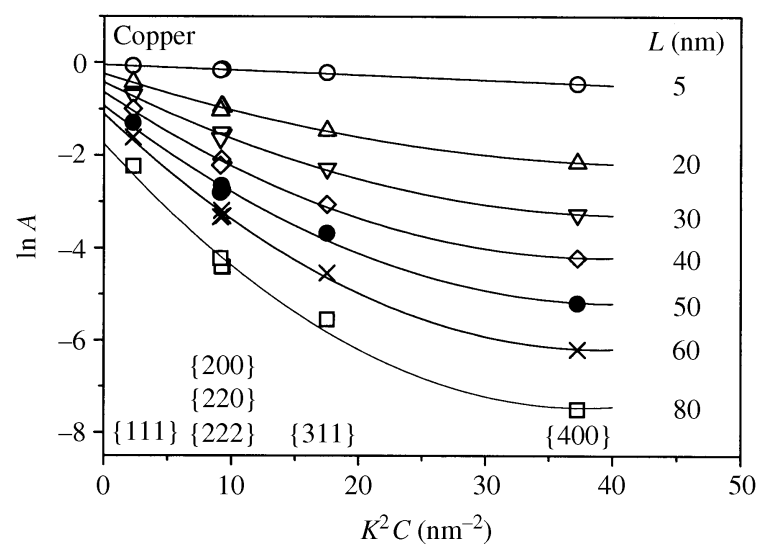

Figure 2

The modified Warren-Averbach plot according to equation (4) for copper deformed by equal-channel angular pressing (Valiev et al., 1994). Note that $C$ is a function of $h k l$; see equation (10). median, $m$, and variance, $\sigma$, of the crystallite size distribution functions determined by the WFFC procedure (see \$2.4) are listed in Table 1.

\subsection{Microstructural parameters obtained from the method of whole-profile fitting using the Fourier coefficients (WPFC)}

Here we present the microstructural parameters obtained by using the Fourier coefficients in the whole-profile fitting (WPFC) procedure, as described in $\$ 3$. The length of the Burgers vector and $\bar{C}_{h 00}$ or $\bar{C}_{h k 0}$ are input parameters. The values of these quantities are the same as those calculated for the WFFC procedure above. The measured and the fitted theoretical Fourier transforms are plotted in Figs. 3 and 4 for copper and silicon nitride, respectively. The open circles and the solid lines represent the measured and the fitted theoretical Fourier profiles normalized to unity, respectively. The sum of squared residuals (SSR) was usually between 0.1 and 1 , which is very satisfactory taking into account that the fitting was carried out on about 1500 to 5000 data points. On a Pentium class machine, one iteration lasts less than $1 \mathrm{~s}$ and convergence to $\Delta(\mathrm{SSR}) / \mathrm{SSR}=10^{-9}$ is usually reached after 10 to 50 iterations. Fitting of one set of profiles took usually less than $1 \mathrm{~min}$. Further details of the fitting procedure and the fitting program may be found elsewhere (Ribárik et al., 2001). The median, $m$, and variance, $\sigma$, of the crystallite size distribution, the dislocation densities, $\rho$, and the arrangement parameters, $M$, of the dislocations, obtained for copper and silicon nitride, are listed in Table 1 . It can be concluded that the results determined by the two different procedures, WFFC and WPFC, are in very good correlation.

In order to check the quality of the fitting, the measured physical profiles are compared with the inverse Fourier transform of the fitted Fourier coefficients in Fig. 5. The differences are also shown. The measured (open circles) and fitted (solid lines) profiles of silicon nitride are shown in Fig. 5(a). Three selected profiles are shown in a wider scale in Fig. 5(b). A very good correlation between the two sets of profiles can be observed. In the case of copper, the linear and logarithmic intensity plots in Figs. $5(c)$ and $5(d)$, respectively, show the central and the tail parts of the profiles in more detail. The profiles correspond to a plastically deformed bulk specimen and are intrinsically asymmetric as a result of residual long-range internal stresses (Mughrabi, 1983; Ungár et al., 1984; Groma et al., 1988; Groma \& Székely, 2000). These internal stresses have the most pronounced effect on the 200, 311 and 400 reflections. Since in the WPFC procedure the $a b$ initio Fourier coefficients correspond to symmetrical profiles, the Fourier coefficients corresponding to the measured profiles were also symmetrized by taking their absolute values. The inverse Fourier transformation of the fitted coefficients therefore cannot account for the asymmetries of the measured profiles. The somewhat larger differences in Fig. 5(b) are caused by this intrinsic asymmetry, especially in the case of the 200, 311 and 400 profiles. The handling of intrinsic asymmetries will be included in the further development of the procedure. 
Table 1

The median, $m$, and the variance, $\sigma$, of the crystallite size distribution functions, the densities, $\rho$, and the arrangement parameters, $M$, of dislocations, and the parameters of the dislocation contrast factors, $q$, or $A$ and $B$, obtained for copper and silicon nitride by the two different X-ray diffraction procedures, WFFC and WPFC.

\begin{tabular}{|c|c|c|c|c|c|c|c|}
\hline Sample & Method & $m(\mathrm{~nm})$ & $\sigma$ & $\rho\left(10^{15} \mathrm{~m}^{-2}\right)$ & $M$ & $q$ & $A, B$ \\
\hline Copper & WFFC & $59(5)$ & $0.51(5)$ & $1.6(2)$ & 2.8 & 1.9 & - \\
\hline Copper & WPFC & $62(5)$ & $0.53(4)$ & $1.7(2)$ & 1.7 & 1.84 & - \\
\hline $\mathrm{Si}_{3} \mathrm{~N}_{4}$ & WFFC & $26(3)$ & $0.54(5)$ & $0.77(8)$ & 2.5 & - & $3.33,-1.78$ \\
\hline $\mathrm{Si}_{3} \mathrm{~N}_{4}$ & WPFC & $20(3)$ & $0.65(5)$ & $0.75(8)$ & 2.1 & - & $3.54,-1.93$ \\
\hline
\end{tabular}

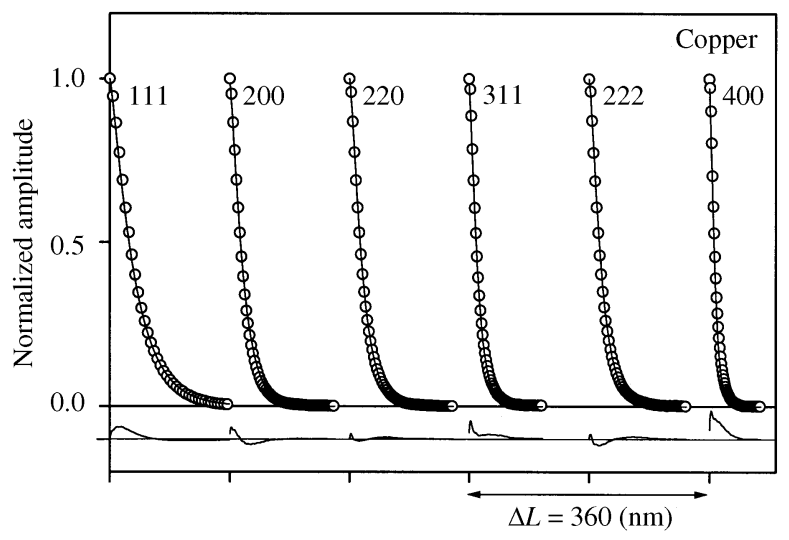

Figure 3

The measured (open circles) and the fitted theoretical (solid line) Fourier coefficients as a function of $L$ for the copper specimen. The differences between the measured and fitted values are also shown, in the lower part of the figure. The scaling of the differences is the same as in the main part of the figure. The indices of the reflections are also indicated.

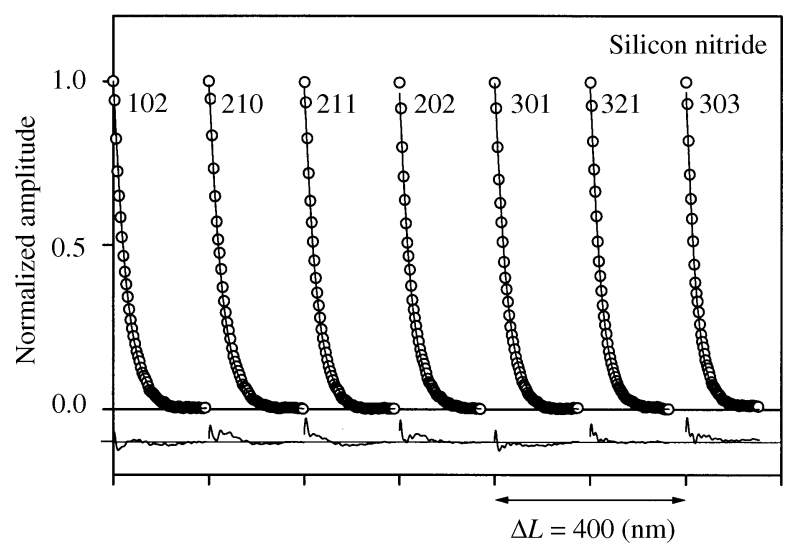

Figure 4

The measured (open circles) and the fitted theoretical (solid line) Fourier coefficients of $L$ for silicon nitride. The differences between the measured and fitted values are also shown, in the lower part of the figure. The scaling of the differences is the same as in the main part of the figure. The indices of the reflections are also indicated.

\subsection{Comparison of the X-ray results with the TEM micro- structure}

The crystallite size distributions, $f(x)$, obtained by X-ray analysis are compared with size distributions determined from TEM micrographs for the silicon nitride loose powder and the plastically deformed bulk copper specimens. Typical TEM micrographs of the two specimens are shown in Figs. 6 and 7. In the TEM micrographs, the crystallite sizes were determined by the usual method of random line section.
For silicon nitride, about 300 particles were measured at random in different areas of the micrographs and are shown as a bar graph in Fig. 8. The crystallite size distribution density function, $f(x)$, obtained by the WFFC method, is shown by a solid line in the same figure. The agreement between the bar diagram and the size distribution function is very good. The small quantitative differences between the X-ray and the TEM results probably arise from the fact that the bar diagram was obtained from a relatively small number of particles. A formidably greater effort would be needed in order to increase the number of particles for counting in TEM micrographs. Estimating the volume illuminated by X-rays and the fraction of crystallites reflecting in the correct direction, the number of crystals contributing to the X-ray measurements is found to be at least five orders of magnitude larger than in the TEM investigations. The good qualitative and quantitative agreement between the size distributions determined by TEM and $\mathrm{X}$-ray analysis for silicon nitride indicates that (i) the particles in the powder are single crystals, i.e. for this powder the phrases 'crystallite' and 'particle' can be used in the same sense, (ii) the size distribution is log-normal, in accordance with observations of many nanocrystalline materials by other authors (Krill \& Birringer, 1998; Terwilliger \& Chiang, 1995; Ungár, Borbély et al., 1999), and (iii) the X-ray procedures described in $\$ \$ 2$ and 3 yield the size distribution in good agreement with direct observations. The area-weighted average crystallite size $\left(\langle x\rangle_{\text {area }}\right)$ of silicon nitride calculated from equation (17) is $58 \mathrm{~nm}$. This value agrees well with the area-weighted average particle size of the powder determined from the specific surface area, $t=71 \mathrm{~nm}$.

In the case of the bulk copper specimen, contour maps were first drawn around the assumed crystallites. A typical firstapproximation contour map is shown in Fig. 7(b) and the corresponding bar graph is shown in Fig. 9 (open squares). The crystallite size distribution density function, $f(x)$, obtained by the WFFC method is shown by a solid line in the same figure. The open squares, annotated as TEM (gross) in Fig. 9, correspond to considerably larger crystallites than the X-ray size distribution. A more careful evaluation of the TEM micrograph in Fig. 7(a) shows that there are large areas not in contrast, which is typical for TEM micrographs of bulk material. By tilting the specimen in the electron microscope, different areas come into contrast or go out of contrast. The contour of a large area out of contrast is shown in Fig. 7(c). On the other hand, some areas are in excellent contrast, for example the grain denoted by $A$ in Fig. 7(b). The contour map has been refined by selecting a large number of regions that 


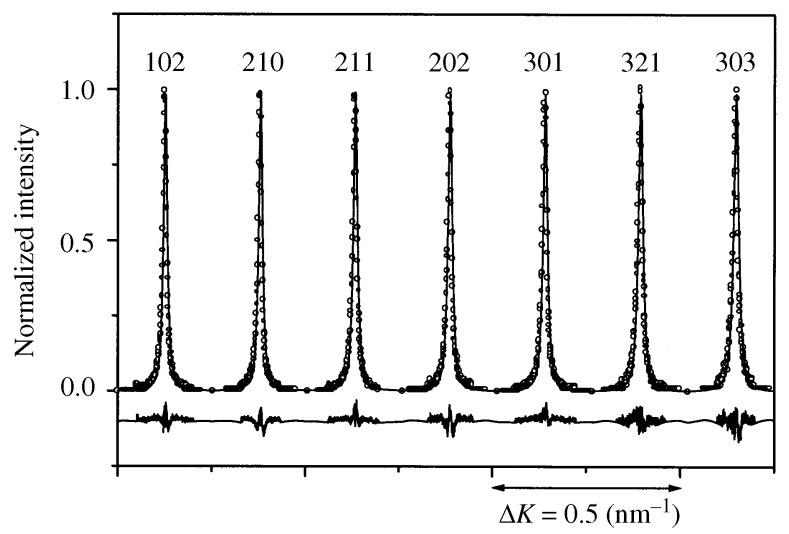

(a)

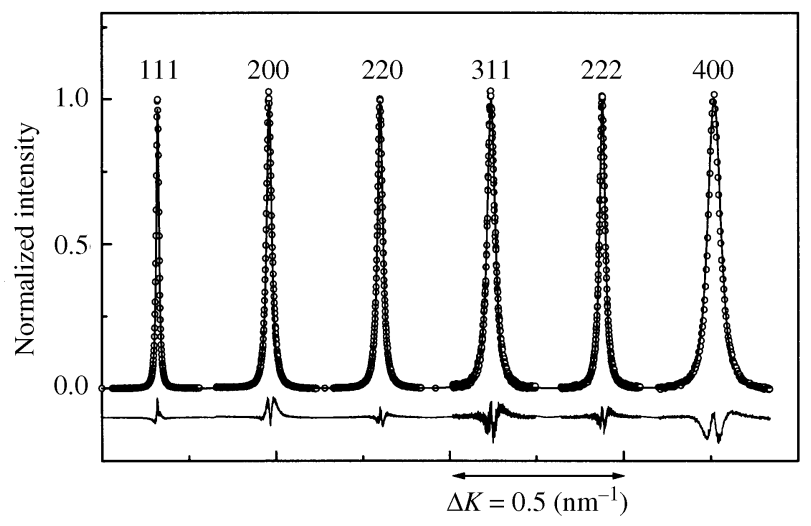

(c)

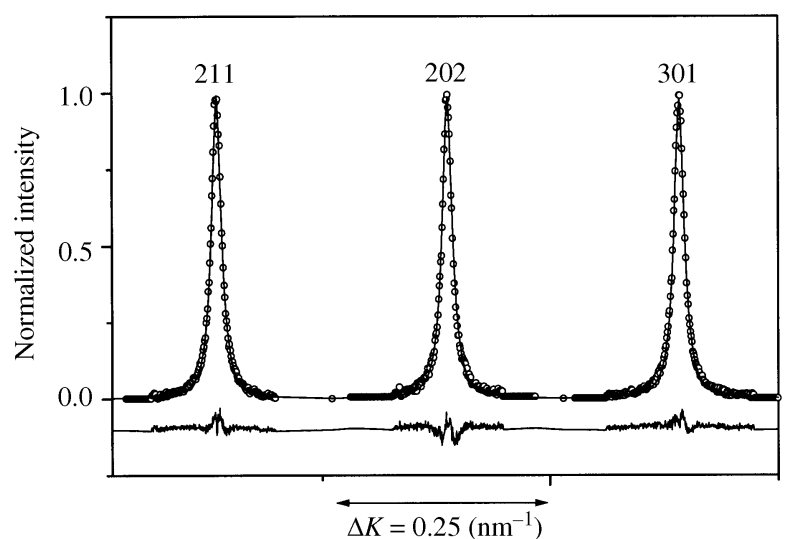

(b)

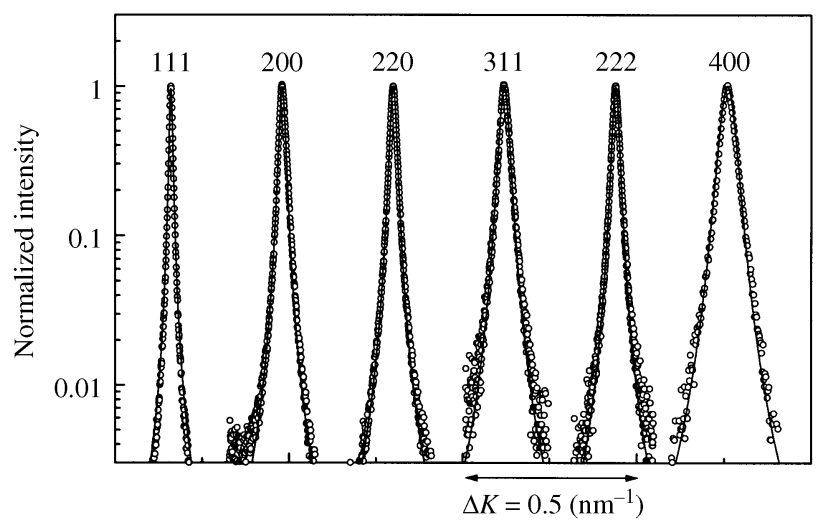

$(d)$

Figure 5

The measured intensity profiles (open circles) and the inverse Fourier transform of the fitted Fourier coefficients (solid lines) for silicon nitride, $(a),(b)$, and for the copper specimen, $(c)$. Three selected profiles of silicon nitride with a wider scale are shown in $(b)$. The profiles corresponding to copper are shown in logarithmic scale in $(d)$. The differences between the measured and fitted intensity values are also shown, in the lower parts of the linear-scale plots $(a),(b)$ and $(c)$. The scaling of the differences is the same as in the main part of the figure. The relatively larger differences in the case of copper are caused by intrinsic asymmetries of the profiles; for details see $\$ 5.2$.

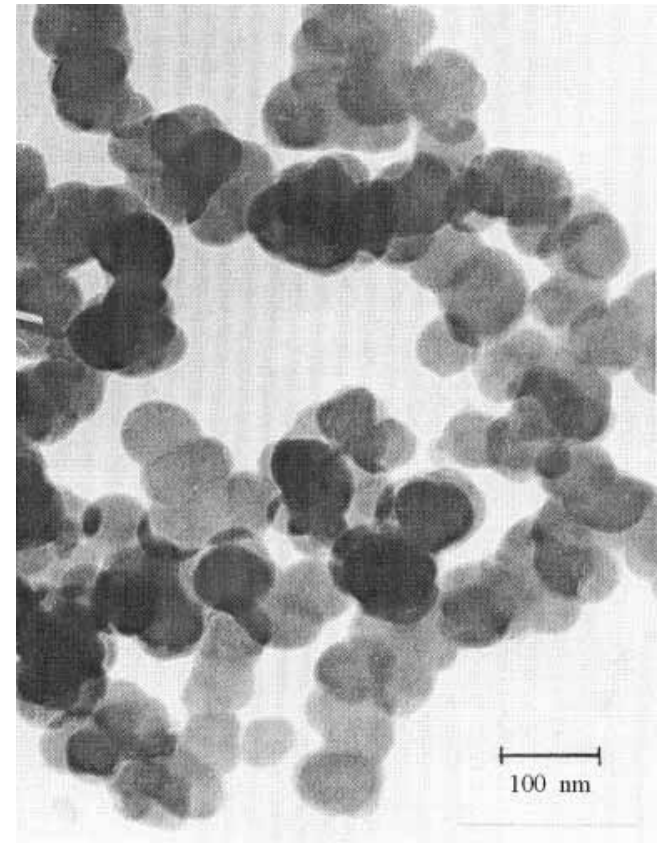

Figure 6

TEM micrograph of the silicon nitride ceramic powder. are in good contrast, using several micrographs. A typical example is shown in Fig. $7(d)$. The size distribution corresponding to the refined contour maps is shown as a bar graph in Fig. 9 and is denoted as TEM (fine).

In a bulk specimen, like the copper specimen investigated here, there is a hierarchy of length scales (Hughes \& Hansen, 1991; GilSevillano, 2001); in sequence of decreasing order: (i) grains, (ii) subgrains, (iii) cell blocks, (iv) dislocation cells, (v) cell interiors, (vi) cell boundaries and (vii) distances between dislocations. (Note that this hierarchy becomes more complicated for bulk materials with different phases, e.g. in alloys containing precipitates or in composites.) The misorientation between the different units of the microstructure can vary from zero through small angles to large angles. In X-ray diffraction, crystallite diameter is equivalent to the size of a domain that is separated from the surroundings by a small misorientation, typically one or two degrees. The contour map in Fig. $7(b)$ is produced by grains, the largest unit in the microstructure. All other units, from subgrains down to cell boundaries, can have very different misorientations, ranging from a few degrees to any large value. It is up to the experimenter to determine which unit the X-ray coherence length 


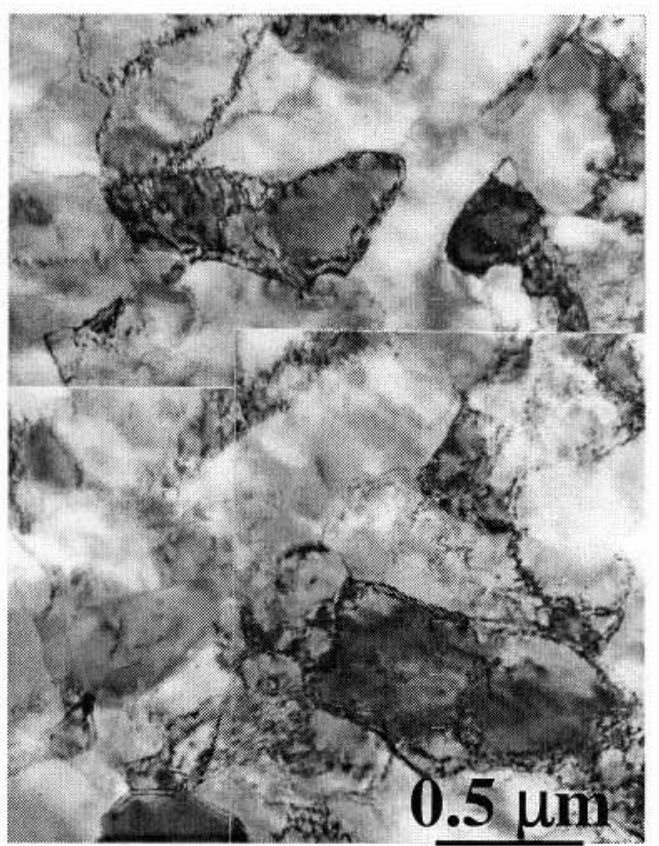

(a)

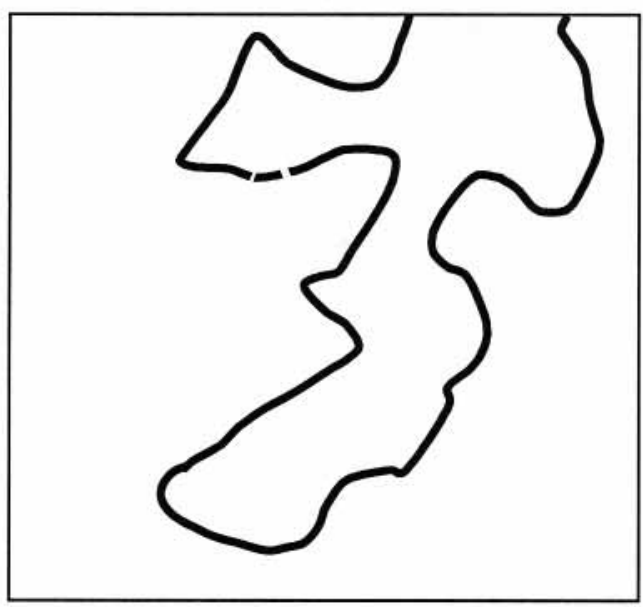

$(c)$

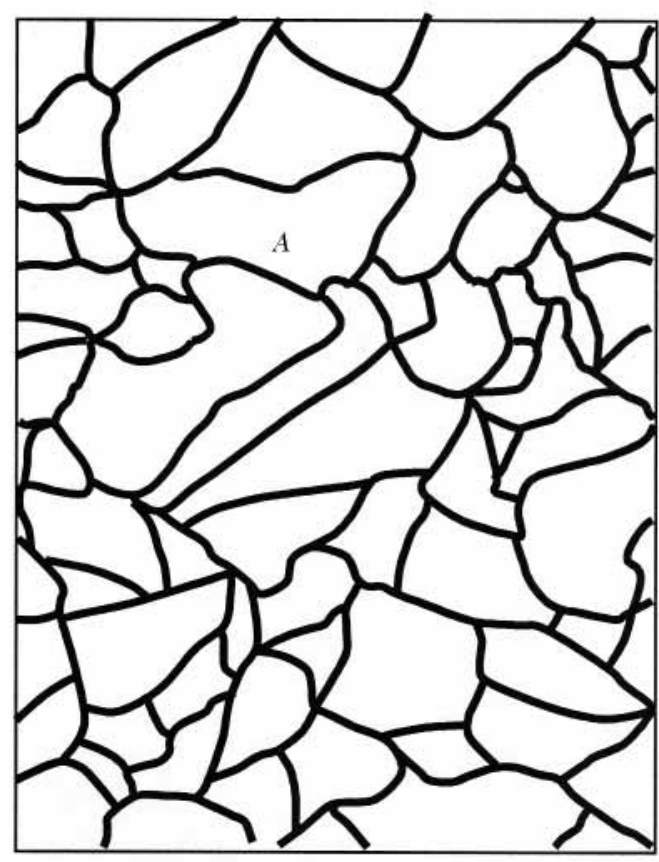

(b)

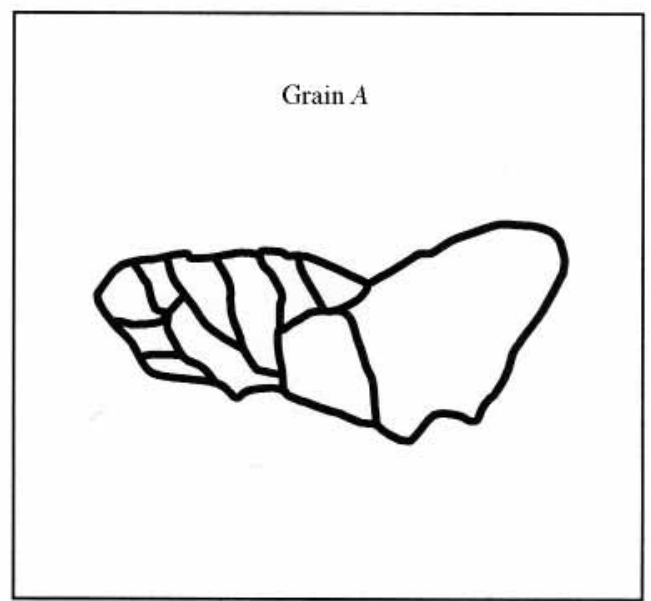

$(d)$

\section{Figure 7}

TEM micrograph of the copper specimen. The lines in $(b)$ represent the contours of the large grains in the micrograph $(a)$. The region out of contrast in the micrograph $(a)$ is shown in $(c) .(d)$ shows grain $A$ divided into smaller subgrains.

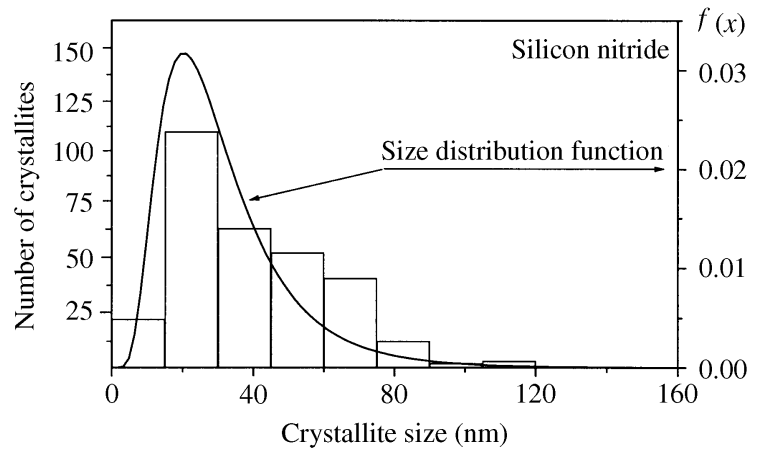

Figure 8

Bar diagram of the crystallite size distribution obtained from TEM micrographs and the size distribution density function, $f(x)$ (solid line), determined by X-ray analysis, for the silicon nitride ceramic powder. corresponds to. For this reason, TEM micrographs are very helpful and almost mandatory for the correct interpretation of $\mathrm{X}$-ray crystallite size distribution in the case of bulk material.

In the copper specimen investigated here, the average dislocation distance is $\rho^{-1 / 2}=36 \mathrm{~nm}$. The median, the volume-, area- and arithmetic-average crystallite size values [see equations (17), (18) and (19)] are 59, 147, 113 and $67 \mathrm{~nm}$, respectively. All crystallite size values are two to six times larger than the average dislocation distance, indicating that the coherent domain size is definitely different from the dislocation distance. A single dislocation does not destroy the coherence of scattering, in agreement with many earlier results (Wilkens, 1988). The present results show that the size distribution obtained from X-ray diffraction is closer to the 


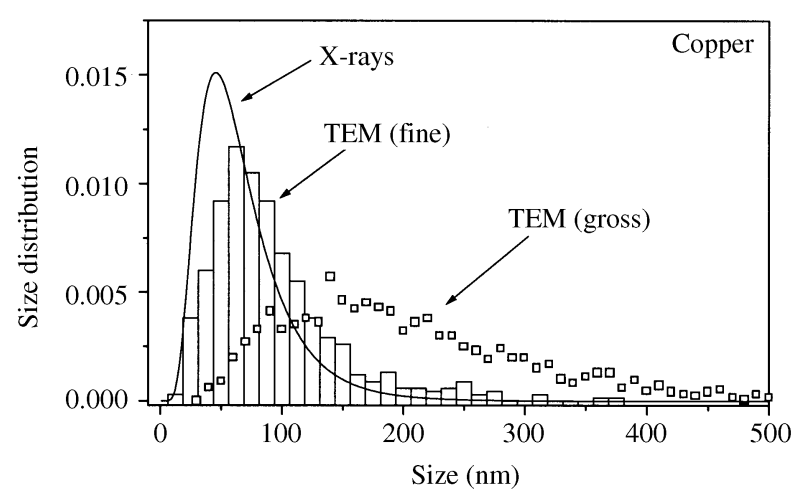

Figure 9

The two size distributions obtained by TEM for the large grains (open squares) and the smaller subgrains (bar diagram) and the size distribution density function, $f(x)$ (solid line), determined by X-ray analysis, for the copper sample.

subgrain size distribution determined from TEM than to classical large-grain size distribution. Obviously, the X-ray and TEM size distributions approach each other as the crystallite size decreases. This is especially true for nanocrystalline materials, irrespective of powder or bulk, as can be seen for the silicon nitride powder here or in previous works on ballmilled and bulk materials (Révész et al., 1996; Ungár et al., 1998; Gubicza et al., 2000).

\section{Conclusions}

Two different procedures are presented to obtain parameters of the microstructure of crystalline materials by diffraction peak profile analysis. One is based on the FWHM, the integral breadths and the first few Fourier coefficients of the profiles. The other one is based on fitting ab initio physical functions to the Fourier transform of the measured profiles.

In both procedures, strain anisotropy is accounted for by the dislocation model of the mean square strain. In cubic or hexagonal crystals, the average dislocation contrast factors are described by two or three parameters, respectively. One or two of these parameters in cubic or hexagonal crystals, respectively, are obtained as a result of the fitting procedure.

By scaling the FWHM, the integral breadths and the Fourier coefficients by the dislocation contrast factors, the strain and size parts of peak broadening can be well and straightforwardly separated from each other, enabling the reliable determination of the apparent size parameters.

It has been shown that the crystallite size distribution can be determined either from the apparent size parameters or from the whole-profile fitting procedure assuming spherical shape and log-normal size distribution of the crystallites.

Although the apparent size parameter corresponding to the FWHM has no direct physical meaning, its inclusion in the determination of the crystallite size distribution decreases the sensitivity of the procedure to the accuracy of the determination of the background.

The Fourier transform of the theoretical size profiles has been derived in a closed form, enabling a convenient and fast fitting procedure.
In the case of spherical crystallites and the absence of stacking faults, the microstructures are characterized by five or six parameters: the median and the variance of the size distribution, the density and the arrangement parameter of dislocations, and one or two parameters for the dislocation contrast factors in cubic or hexagonal crystals, respectively. In the two procedures these are the only fitting parameters.

The two different methods were applied to determine the crystallite size distribution and the dislocation structure in a severely deformed bulk copper sample and a loose powdered silicon nitride specimen. Good correlation between the microstructural parameters provided by the two different methods of diffraction profile analysis, WFFC and WPFC, is observed.

In the case of silicon nitride, the crystallite size distributions obtained by the two different methods are in excellent agreement with the TEM results. The area-weighted mean crystallite size obtained by X-ray analysis is in good agreement with the area-weighted mean particle size calculated from the specific surface area provided by the method of BET. From this, it is concluded that the silicon nitride particles are monocrystalline.

The TEM micrographs of the bulk copper specimen were evaluated with regards to (i) the grains separated by the strongest contours and (ii) the subgrains surrounded by weaker contours. The results of the second evaluation are in good correlation with the crystallite size distribution determined by X-ray analysis. From this it is concluded that in plastically deformed bulk materials, the coherently scattering domains are closer to subgrains or dislocation cells than to crystallographic grains.

The authors are indebted to Dr Katalin Tasnády for the TEM measurements. The authors are grateful for the financial support of the Hungarian Scientific Research Fund, OTKA, Grant Nos. T031786, T029701, D29339 and AKP 98-25.

\section{References}

Armstrong, N. \& Kalceff, W. (1999). J. Appl. Cryst. 32, 600-613.

Barabash, R. I. \& Klimanek, P. (1999). J. Appl. Cryst. 32, 1050-1059.

Berkum, J. G. M. van, Vermuelen, A. C., Delhez, R., de Keijser, Th.

H. \& Mittemeijer, E. J. (1994). J. Appl. Cryst. 27, 345-357.

Bertaut, E. F. (1950). Acta Cryst. 3, 14-18.

Borbély, A., Driver, J. H. \& Ungár, T. (2000). Acta Mater. 48, 20052016.

Caglioti, G., Paoletti, A. \& Ricci, F. P. (1958). Nucl. Instrum. 3, $223-$ 228.

Černý, R., Joubert, J. M., Latroche, M., Percheron-Guegan, A. \& Yvon, K. (2000). J. Appl. Cryst. 33, 997-1005.

Chatterjee, P. \& Sen Gupta, S. P. (1999). J. Appl. Cryst. 32, 1060-1068. Cheary, R. W., Dooryhee, E., Lynch, P., Armstrong, N. \& Dligatch, S. (2000). J. Appl. Cryst. 33, 1271-1283.

Dinnebier, R. E., Von Dreele, R., Stephens, P. W., Jelonek, S. \& Sieler, J. (1999). J. Appl. Cryst. 32, 761-769.

GilSevillano, J. (2001). Mater. Sci. Eng. A. In the press.

Groma, I. (1998). Phys. Rev. B, 57, 7535-7542.

Groma, I. \& Székely, F. (2000). J. Appl. Cryst. 33, 1329-1334.

Groma, I., Ungár, T. \& Wilkens, M. (1988). J. Appl. Cryst. 21, 47-53. 
Gubicza, J., Ribárik, G., Goren-Muginstein, G. R., Rosen, A. R. \& Ungár, T. (2001). Mater. Sci. Eng. A. In the press.

Gubicza J., Szépvölgyi, J., Mohai, I., Zsoldos, L. \& Ungár, T. (2000). Mater. Sci. Eng. A, 280, 263-269.

Guinier, A. (1963). X-ray Diffraction. San Francisco: Freeman.

Hecker, M., Thiele, E. \& Holste, C. (1997). Mater. Sci. Eng. A, 234236, 806-809.

Hinds, W. C. (1982). Aerosol Technology: Properties, Behavior and Measurement of Airbone Particles. New York: Wiley.

Hughes, D. A. \& Hansen, N. (1991). Mater. Sci. Technol. 7, 544-553.

Kamminga, J. D. \& Delhez, R. (2000). J. Appl. Cryst. 33, 1122-1127.

Klimanek, P. \& Kužel, R. Jr (1988). J. Appl. Cryst. 21, 59-66.

Klug, H. P. \& Alexander, L. E. (1974). X-ray Diffraction Procedures for Polycrystalline and Amorphous Materials, 2nd ed. New York: Wiley.

Krill, C. E. \& Birringer, R. (1998). Philos. Mag. A, 77, 621-640.

Krivoglaz, M. A. (1969). Theory of X-ray and Thermal Neutron Scattering by Real Crystals. New York: Plenum Press.

Krivoglaz, M. A. (1996). X-ray and Neutron Diffraction in Nonideal Crystals. Berlin: Springer-Verlag.

Kužel, R. Jr \& Klimanek, P. (1988). J. Appl. Cryst. 21, 363-368.

Langford, J. I., Louer, D. \& Scardi, P. (2000). J. Appl. Cryst. 33, 964 974.

Le Bail, A. \& Jouanneaux, A. (1997). J. Appl. Cryst. 30, 265-271.

Levine, L. E. \& Thomson, R. (1997). Acta Cryst. A53, 590-602.

Lippenca, B. C. \& Hermanns, M. A. (1961). Powder Met. 7, 66-74.

Louër, D., Auffredic, J. P., Langford, J. I., Ciosmak, D. \& Niepce, J. C. (1983). J. Appl. Cryst. 16, 183-191.

Mughrabi, H. (1983). Acta Metall. 31, 1367-1379.

Rajan, K. \& Sajgalik, P. J. (1997). J. Am. Ceram. Soc. 17, 1093-1097.

Révész, Á., Ungár, T., Borbély, A. \& Lendvai, J. (1996). Nanostruct. Mater. 7, 779-788.

Ribárik, G., Ungár, T. \& Gubicza, J. (2001). J. Appl. Cryst. Submitted. Popa, N. C. (1998). J. Appl. Cryst. 31, 176-180.

Rand, M., Langford, J. I. \& Abell, J. S. (1993). Philos. Mag. B, 68, 1728.

Rao, S. \& Houska, C. R. (1986). Acta Cryst. A42, 6-13.

Scardi, P. \& Leoni, M. (1999). J. Appl. Cryst. 32, 671-682.

Stephens, P. W. (1999). J. Appl. Cryst. 32, 281-289.

Stokes, A. R. (1948). Proc. Phys. Soc. London, 61, 382-393.

Terwilliger, Ch. D. \& Chiang, Y. M. (1995). Acta Metall. Mater. 43, 319-328.
Trinkaus, H. (1972). Phys. Status Solidi B, 51, 307-319.

Ungár, T. \& Borbély, A. (1996). Appl. Phys. Lett. 69, 3173-3175.

Ungár, T., Borbély, A., Goren-Muginstein, G. R., Berger, S. \& Rosen, A. R. (1999). Nanostruct. Mater. 11, 103-113.

Ungár, T., Dragomir, I., Révész, Á. \& Borbély, A. (1999). J. Appl. Cryst. 32, 992-1002.

Ungár, T., Gubicza, J., Ribárik, G. \& Zerda, T. W. (2001). Proceedings of the MRS Fall Meeting, 2000, Boston. MRS Symp. Vol. 661, KK9.2.1-6.

Ungár, T., Leoni, M. \& Scardi, P. (1999). J. Appl. Cryst. 32, 290-295.

Ungár, T., Mughrabi, H., Rönnpagel, D. \& Wilkens, M. (1984). Acta Metall. 32, 333-342.

Ungár, T., Mughrabi, H. \& Wilkens, M. (1982). Acta Metall. 30, 18611867.

Ungár, T., Ott, S., Sanders, P., Borbély, A. \& Weertman, J. R. (1998). Acta Mater. 46, 3693-3699.

Ungár, T. \& Tichy, G. (1999). Phys. Status Solidi A, 171, 425-434.

Valiev, R. Z., Ishlamgaliev, R. K. \& Alexandrov, I. V. (2000). Prog. Mater. Sci. 45, 103-189.

Valiev, R. Z., Kozlov, E. V., Ivanov, Yu. F., Lian, J., Nazarov, A. A. \& Baudelet, B. (1994). Acta Metall. Mater. 42, 2467-2476.

Wang, Ch.-M., Pan, X., Ruehle, M., Riley, F. L. \& Mitomo, M. J. (1996). Mater. Sci. 31, 5281-5298.

Warren, B. E. (1959). Prog. Met. Phys. 8, 147-202.

Warren, B. E. \& Averbach, B. L. (1950). J. Appl. Phys. 21, 595-595.

Wilkens, M. (1970a). Phys. Status Solidi. A, 2, 359-370.

Wilkens, M. (1970b). Fundamental Aspects of Dislocation Theory, Vol. II, edited by J. A. Simmons, R. de It \& R. Bullough, pp. 11951221. Natl Bur. Stand. (US) Spec. Publ. No. 317, Washington, DC. Wilkens, M. (1987). Phys. Status Solidi A, 104, K1-K6.

Wilkens, M. (1988). Proceedings of the 8th International Conference on the Strength Metal Alloys (ICSMA 8), Tampere, Finland, edited by P. O. Kettunen, T. K. Lepistö \& M. E. Lehtonen, pp. 47-152. Oxford: Pergamon Press.

Wilkens, M. \& Eckert, H. (1964). Z. Naturforsch. Teil A, 19, 459-470. Williamson, G. K. \& Hall, W. H. (1953). Acta Metall. 1, 22-31.

Wilson, A. J. C. (1962). X-ray Optics. London: Methuen.

Zehetbauer, M. (1993). Acta Metall. Mater. 41, 589-599.

Zehetbauer, M. \& Seumer, V. (1993). Acta Metall. Mater. 41, 577-588.

Zehetbauer, M., Ungár, T., Kral, R., Borbély, A., Schafler, E., Ortner, B., Amenitsch, H. \& Bernstorff, S. (1999). Acta Mater. 47, 10531061. 